\title{
Diversité et structure des groupements ligneux du parc national d'Arly (Est du Burkina Faso)
}

\author{
Oumarou Ouedraogo, Adjima Thiombiano, Karen Hahn-Hadjali et Sita Guinko
}

Received: 07.01.2008; accepted: 31.01 .2008

\begin{abstract}
RÈSUMÈ: La présente étude menée sur la végétation ligneuse du parc national d'Arly a pour objectif de caractériser la diversité et la structure des différents groupements végétaux en relation avec les conditions écologiques. 243 relevés phytosociologiques ont été réalisés. Ces relevés font ressortir 109 espèces ligneuses et 10 groupements par la méthode d'analyse DCA. La diversité des groupements végétaux a été caractérisée par le calcul des indices de diversité. Pour chaque groupement ligneux, 10 relevés ont été choisis de manière aléatoire pour des mesures dendrométriques. Les individus dont le diamètre à $1,3 \mathrm{~m}$ du sol est supérieur à $5 \mathrm{~cm}$ ont été mesurés. La structure des groupements a été décrite à travers la répartition des individus en classe de diamètre, le calcul de la densité et de la surface terrière. La relation entre la diversité et la structure des groupements a été établie sur la base d'analyse de corrélation. Les groupements ligneux distingués sont répartis suivant le gradient topographique. L'analyse de la structure démographique en classe de diamètre montre que les peuplements des différents groupements végétaux sont stables, ce qui traduit leur adaptation aux facteurs écologiques stationnelles telles que le feu et les conditions de sol. Les forêts claires à Tamarindus indica et Combretum paniculatum et les forêts galeries à Vitex chrysocarpa et Garcinia livingstonei sont les formations les plus denses et les groupements ligneux les plus diversifiés. A l'opposé, les savanes arbustives clairsemées à Euphorbia sudanica et Combretum niorense et celles à Acacia sieberiana sont moins denses et constituent les groupements les plus pauvres en espèces. Ainsi, il existe une relation entre la diversité et la structure des groupements des formations ligneuses. Les conditions édaphiques et topographiques occupées par ces groupements expliquent à la fois leur diversité et leur structure.
\end{abstract}

Mots clés: groupements ligneux, relevés, stabilité, sol

\section{Diversity and Structure of the Woody Plant Communities in the National Park of Arly}

SUMMARY: The diversity and structure of woody vegetation were studied in the national park of Arly in relation to the ecological conditions. After vegetation classification by DCA analysis, diversity parameters, density, basal area and size class distribution of each plant community were determined. We characterised 10 plant communities whose distribution is governed by a topo-edaphic gradient. The patterns of size class distribution show that the stands of all plant communities are in a stable situation. This result shows the adaptation of the vegetation units to the local environmental conditions as fire regime and soil properties. Woodlands of Tamarindus indica and Combretum paniculatum and riparian forests of Vitex chrysocarpa and Garcinia livingstonei possess the high est density and the highest diversity of tree species. In contrary, wooded grasslands of Euphorbia sudanica and Combretum niorense and humid wooded grasslands of Acacia sieberiana are very poor in species and their density is low. These results supported by correlation analysis show a significant relation between diversity and structure. Topo-edaphic conditions are one of the principal environmental factors which drive the diversity and the structure of plant communities.

Key words: woody plant community, relevés, stability, soil

\section{INTRODUCTION}

Le rôle principal des aires protégées étant la conservation de la biodiversité, la connaissance de la dynamique des écosystèmes qu'elles renferment devient une préoccupation fondamentale pour tous les acteurs impliqués dans leur gestion. $\mathrm{Au}$ plan international, l'attention soutenue de la communauté internationale sur la biodiversité a favorisé une multitude d'études montrant la nécessité de conserver celle-ci en relation avec les ressources et les aires disponibles (JON et JoN 1997). Cependant, il est important de constater que la connaissance sur la flore des réserves de l'Est du Burkina Faso reste sommaire. Ce manque de données sur la végétation compromet tout suivi écologique efficace et durable de la biodiversité.

Le parc national d'Arly fait partie des unités de protection et de conservation de la biodiversité de l'Est du Burkina Faso.
Il renferme une physiographie très hétérogène à laquelle s'associe une diversité d'écosystèmes. Ses atouts naturels lui confèrent un site par excellence de conservation. En effet, face au manque d'espaces à vocations agropastorales et à la raréfaction de la biodiversité dans les espaces anthropisés, le parc à l'instar des autres aires protégées est sans cesse convoité par les populations riveraines pour leurs besoins divers tels que les alimentations humaine et du bétail, les bésoins énergétiques, la construction, la pharmacopée traditionnelle (UICN 2004). C'est la composante végétale de ces réserves qui est quantitativement plus affectées par les riverains et aussi les éleveurs transhumants. Dans ces complexes de conservation et de protection comme dans tout autre paysage, la végétation joue un rôle important et multidirectionnel dans le contrôle de la biodiversité (COUTERON et KOKOU 1995, JÜRGENS et al. 2003). Elle est une résultante de faits 
environnementaux et donc un bon indicateur pour apprécier la stabilité des écosystèmes. Un des principaux objectifs d'étude en écologie forestière est la déduction des changements passés et la prédiction des futurs, dans la composition spécifique (CONDIT et al. 1998). Aussi, les investigations sur la structure et la diversité sont des éléments indicateurs qui permettent d'analyser les tendances d'évolution qualitative et quantitative de la végétation (OUÉDRAOGO 2006).

La présente étude a pour objectifs de déterminer d'abord les différents groupements végétaux ligneux, ensuite d'étudier leur diversité et leur structure et enfin d'analyser s'il y a des liens entre les paramètres de diversité et de structure en reponse aux facteurs écologiques.

\section{Milieu d'Étude}

Le parc national d'Arly s'étend sur une superficie de 120.000 ha entre les latitudes $11^{\circ} 75^{\prime}$ à $11^{\circ} 24^{\prime}$ Nord et les longitudes $01^{\circ} 14^{\prime}$ à $01^{\circ} 78^{\prime}$ Est. Le climat est marqué par l'alternance de deux saisons. Une saison pluvieuse débutant d'avril au mois d'octobre et une saison sèche de novembre à mars. La moyenne annuelle des précipitations couvrant la période de 1975 à 2005 est de 856,2 $\mathrm{mm}$. Selon le découpage phytogéographique du Burkina Faso réalisé par GUINKO (1984), le parc d'Arly appartenait au secteur phytogéographique sud soudanien dans sa limite nord. En tenant compte de la moyenne annuelle des précipitations des trentaines dernières années (1975-2005) et du décalage des isohyètes survenus dans ces dernières décennies, le parc appartient actuellement au secteur phytogéographique nord soudanien où la pluviométrie en année normale varie entre $750 \mathrm{~mm}$ et $1000 \mathrm{~mm}$ (GuINKo 1984). Les sols comprennent les lithosols sur cuirasse ferrugineuse, les sols gravillonnaires et les sols ferrugineux léssivés sur matériaux argilo-sableux, les sols hydromorphes vertiques, les sols minéraux bruts sur grès (BOULET et LEPRUN 1969).

\section{MÉthodologie}

\subsection{Récolte de données floristiques et analyse}

L'approche phytosociologique de Braun Blanquet définie par GuINOCHET (1973) a été utilisée. Cette méthode est très actuelle dans les études de la végétation en Afrique de l'Ouest (Adomou 2005, Thiombiano 2005, OuOba 2006). Ainsi 243 relevés phytosociologiques ont été réalisés sur tous les faciès de végétations homogènes suivant les différentes unités géomorphologiques pendant la saison pluvieuse où la végétation est luxuriante facilitant la distinction des espèces sur le terrain. En se basant sur certains travaux ménés sur la végétation des savanes de l'Afrique de l'Ouest (NATta 2003, Wala 2004, Thiombiano 2005), les superficies des relevés étaient de $900 \mathrm{~m}^{2}$ pour les formations savanicoles et les forêts claires et $500 \mathrm{~m}^{2}$ pour les formations galeries.

Les données brutes constituant une matrice de 243 relevés et 109 espèces ligneuses ont été soumises à l'analyse factorielle par la méthode de DCA (Detrended Correspondence Analysis) dans le logiciel CAP version 2.0. Il existe plusieurs méthodes d'analyse numérique des relevés et chacune d'entre elles a une part de subjectivité (FEOLI et al. 2006). Néanmoins, parmi les méthodes indirectes d'ordination, la DCA est plus performante car non sujette à l'effet d'arc et de compression des données (KÉvin et al. 2000). Les groupements ligneux sont nommés par une ou deux espèces caractéristiques.

\subsection{Etude de la diversité des groupements ligneux}

Les paramètres d'étude de la diversité des communautés végétales les plus utilisés sont: la richesse spécifique totale, la richesse spécifique moyenne par relevé, l'indice de Shannon, l'indice de Piélou (Oumorou 2004). Ces différents indices de diversité ont permis de comparer les groupements entre eux et de comprendre la relation qui existe entre la
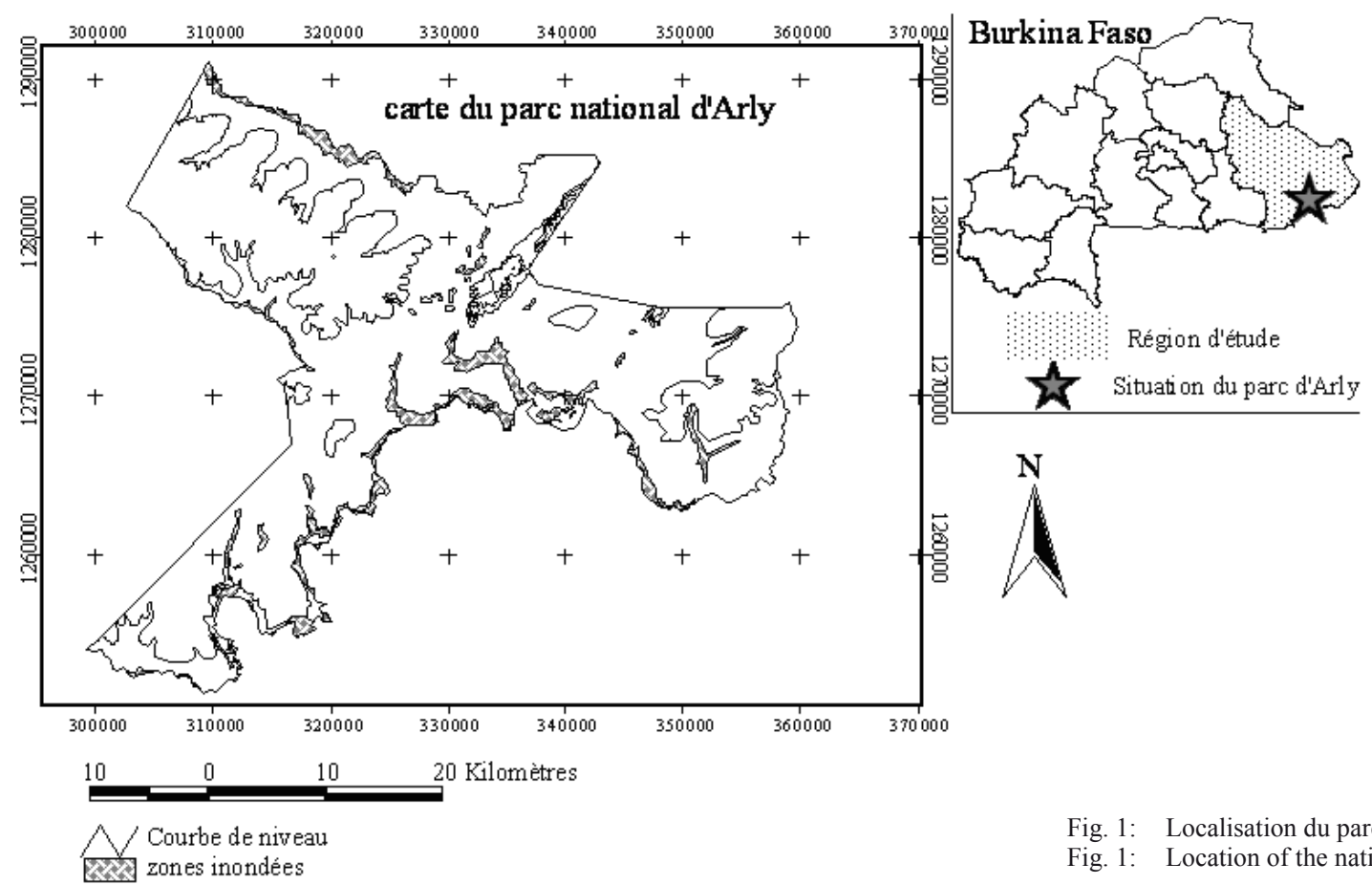

Fig. 1: Localisation du parc national d'Arly

Fig. 1: Location of the national park of Arly 
diversité des communautés végétales en rapport avec leur structure et indirectement avec les conditions écologiques qu'elles colonisent.

\subsection{Relevés dendrométriques et analyse des données structurales}

Pour chaque groupement ligneux, 10 relevés sont choisis de manière aléatoire pour les mesures dendrométriques, soit au total 100 placeaux. La superficie des placeaux reste identique en fonction des types de formation comme indiquée précédemment. A l'intérieur de chaque placeau, le diamètre des individus dont le dbh est supérieur à $5 \mathrm{~cm}$ à $1,30 \mathrm{~m} \mathrm{du}$ sol est mesuré. Lorsque les individus d'une même espèce forment des touffes, seul le plus gros diamètre est pris en compte. Tous les individus dont le dbh est inférieur à $5 \mathrm{~cm}$ sont systématiquement considérés comme faisant partie de la régénération.

Les individus des peuplements ont été répartis en 10 classes de diamètre. Ce sont les classes de 5-10 cm, 10-15 cm, 15 $20 \mathrm{~cm}, 20-25 \mathrm{~cm}, 25-30 \mathrm{~cm}, 30-35 \mathrm{~cm}, 35-40 \mathrm{~cm}, 40-45$ $\mathrm{cm}, 45-50 \mathrm{~cm}, \geq 50 \mathrm{~cm}$. Dans les formations savanicoles du domaine soudanien, beaucoup d'espèces ligneuses n'ont pas un gros diamètre et les individus sont concentrés dans les classes de diamètres inférieures à $50 \mathrm{~cm}$. Dans ce contexte, pour mieux analyser la structure de la végétation, ces 10 classes de diamètre ont été retenues. Ce choix tient aussi compte des travaux antérieurs menées dans les mêmes zones soudaniennes (SAMBOU 2004, GoudiabY 2004, OuÉDRAOGO 2006). Les individus dont les diamètres sont supérieurs ou égal à $50 \mathrm{~cm}$ ont été rassemblés dans une seule classe. Au delà de ce diamètre, le nombre d'individus devient assez rares et pour des besoins de comparaisons et pour percevoir l'influence de certains facteurs environnementaux sur la structure de la végétation, il était nécessaire de réunir ces grands sujets dans une même classe.
La densité de chaque groupement est calculée par le rapport entre le nombre total d'individus mesurés sur le total de surface considérée. Les valeurs de densité sont ensuite converties en nombre d'individus par hectare.

La surface terrière de chaque groupement est calculée à partir de la formule suivante:

$$
\mathrm{G}=\Sigma \pi \mathrm{D}^{2} / 4 \quad \text { avec } \mathrm{D}=\text { diamètre à } 1,3 \mathrm{~m} \text { du sol }
$$

\subsection{Analyse de la relation diversité et structure des groupements et identification des groupes homo- gènes}

Deux test statistiques ont été calculés entre les valeurs des différents paramètres de diversité et de structure à l'aide du logiciel STATISTICA version 4: le test de corrélation de Pearson pour l'étude de la relation diversité-structure et le test LSD de comparaison de moyenne pour la distinction des groupes homogènes. Le niveau de significativité des résultats retenu est de $5 \%$.

\section{RÉsultats}

\subsection{Discrimination des groupements végétaux ligneux}

Les 243 relevés issus du premier traitement se répartissent en deux blocs (fig. 2) le bloc MI renferme tous les relevés exécutés dans les milieux inondables pendant les périodes de pluie et le bloc ME, les relevés des milieux exondés. L'axe 1 d'une longueur de gradient de 9,36 (tableau 1) décrit le gradient majeur et traduit la topographie. Sa combinaison avec l'axe 2 de longueur 4,61 a permis la discrimination des deux blocs. L'axe 2 n'oppose pas clairement à une échelle donnée les deux blocs d'où son interprétation assez difficile. Le nuage de relevés étant diffus pour l'identification des groupements bien distincts, nous avons procédé à une analyse partielle de chaque bloc afin de mieux distinguer ceux ci.

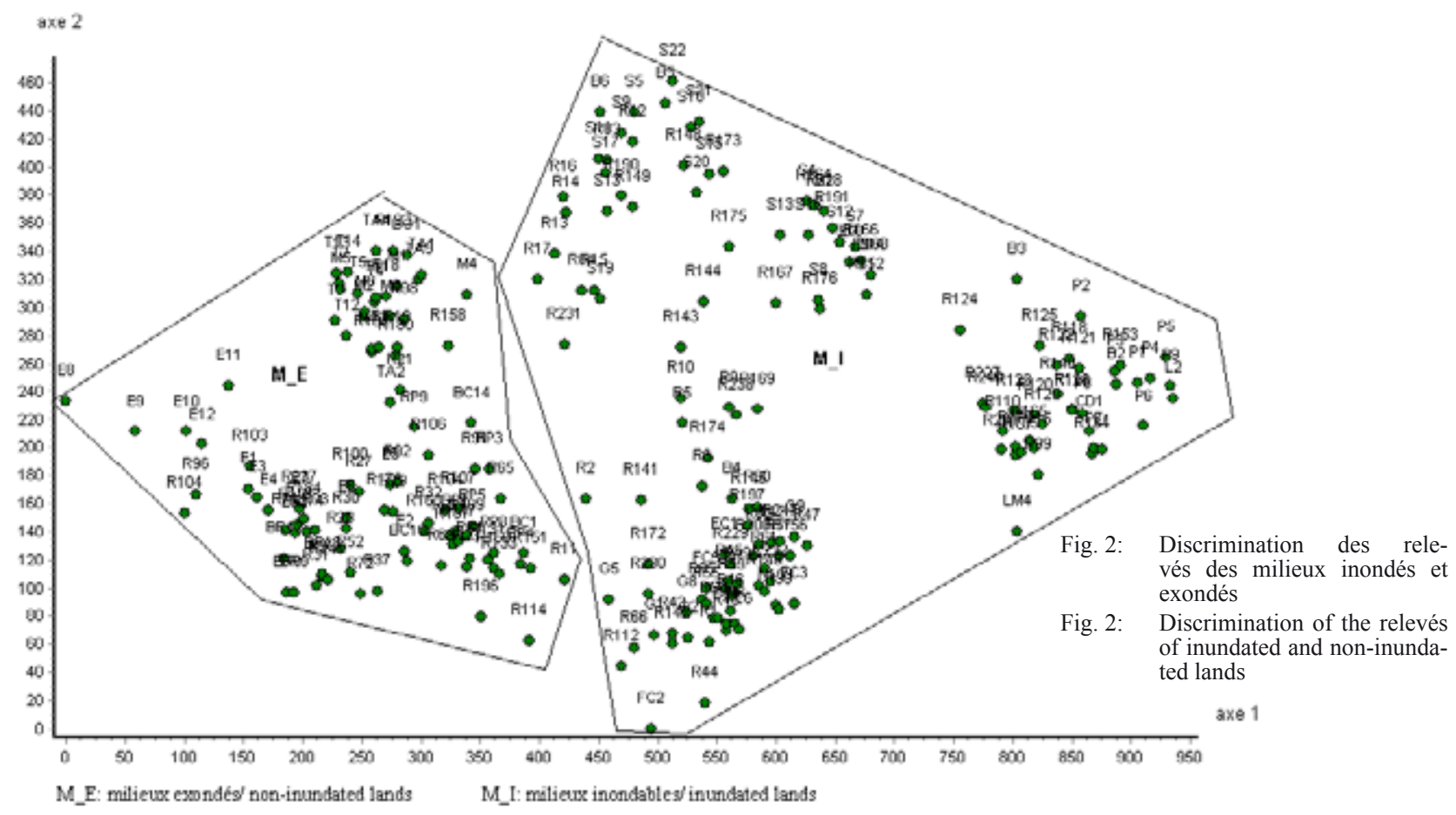


Tableau 1: Longueurs de gradient et valeurs propres

Table 1: Gradient length and Eigenvalues

\begin{tabular}{|c|c|c|c|c|c|c|c|c|c|c|c|c|}
\hline \multirow{4}{*}{$\begin{array}{l}\text { Matrice de données } \\
\text { Axe }\end{array}$} & \multicolumn{4}{|c|}{ Analyse globale } & \multicolumn{8}{|c|}{ Analyse partielle } \\
\hline & & & & & Milieux & inondés & & & milieux & exondés & & \\
\hline & 243 & relevés & et 109 & espèces & 145 & relevés & et $81 \mathrm{e}$ & èces & 97 & relevés et & $69 \mathrm{e}$ & ces \\
\hline & axe 1 & axe 2 & axe 3 & axe 4 & axe 1 & axe 2 & axe 3 & axe 4 & axe 1 & axe 2 & axe 3 & axe 4 \\
\hline Longueur de gradient & 9,4 & 4,6 & 3,9 & 4,4 & 5,9 & 3,8 & 3,1 & 2,8 & 5,5 & 3,4 & 3,1 & 2,9 \\
\hline Valeur propre & 0,9 & 0,5 & 0,4 & 0,3 & 0,8 & 0,4 & 0,3 & 0,2 & 0,7 & 0,4 & 0,3 & 0,2 \\
\hline
\end{tabular}

L'analyse partielle du bloc MI a porté sur une matrice de 145 relevés et de 81 espèces. Cinq groupements ligneux sont distingués (fig. 3). L'axe 1 de variation correspond toujours au gradient topographique car écarte aux deux extrémités les groupements des lits des cours d'eau aux groupements des plaines d'inondations présentant une faible pente. Ainsi de la gauche vers la droite, on a:

- le groupement à Terminalia macroptera et Gardenia ternifolia (Tm_Gt) des plaines à inondation temporaire avec des sols argileux,

- le groupement à Acacia gerrardii et Combretum acuelatum (Ag_Ca) sur zone dépressionnaire à sols vertisoliques,

- le groupement à Acacia sieberiana (As) des plaines alluviales sur sols argileux à période d'inondation prolongée,

- le groupement à Tamarindus indica et Combretum paniculatum (Ti_Cp) sur les axes de drainage et les ravins sur sols argilo-limoneux,

- Le groupement à Vitex chrysocarpa et Garcinia livingstonei (Vc_Gl) colonisant les lits majeurs des cours d'eau sur sol argileux.

L'axe 2 décrit les types de formations et éloigne au mieux les forêts claires à Tamarindus indica et Combretum paniculatum aux savanes arbustives clairsemées à Acacia sieberiana.

L'analyse partielle du bloc ME sur la matrice composée de 97 relevés et 69 espèces a permis également de distinguer 5 groupements (fig. 4) qui sont:

- le groupement à Terminalia avicennioides et Ximenia americana (Ta_Xa) évoluant sur les glacis avec sols sableux profonds,

- le groupement anthropique à Parkia biglobosa et Piliostigma reticulatum $\left(\mathrm{Pb} \_\mathrm{Pr}\right)$ établit dans les moyens glacis sur sols sableux profonds,

- le groupement à Acacia gourmaensis et Acacia macrostachya (Ag_Am) des plateaux et zone de pente sur sols gravillonnaires présentant souvent des affleurements cuirassés,

- le groupement à Burkea africana et Detarium microcarpum (Ba_Dm) colonisant les parties des buttes rocheuses où le substrat est sableux avec des blocs de granites et présentant des anfractuosités importantes,

- le groupement à Euphorbia sudanica et Combretum niorense (Es_Cn) rencontrés sur les parties indurées des buttes rocheuses et sur le plateau de la chaîne gréseuse de montagne du Gobnangou.

Dans ce cas l'axe 1 peut être considéré comme un gradient topographique car oppose le groupement à Euphorbia sudanica et Combretum niorense (Es_Cn) inventorié sur le pla- teau de la chaîne de montagne du Gobnangou et des buttes rocheuses au groupement à Terminalia avicennioides et $X i$ menia americana (Ta_Xa) des moyennes et bas de pentes de glacis.

\subsection{Diversité et structure des groupements ligneux}

Les indices de diversité ainsi que les paramètres de structure varient d'un groupement à l'autre (tableau 2). En considérant l'intervalle de confiance des moyennes, on peut classifier les groupements ligneux pour chaque paramètre en trois classes: les groupements à valeurs faibles, les groupements à valeurs moyennes et ceux à valeurs élevées. Par rapport à la richesse totale d'espèces définissant chaque groupement, le groupement à Terminalia avicennioides et Ximenia americana, et celui à Acacia gourmaensis et Acacia macrostachya totalisent le plus d'espèces avec respectivement 47 et 43 espèces. Les groupements enregistrant moins d'espèces sont le groupement à Acacia sieberiana avec 13 espèces et le groupement à Euphorbia sudanica et Combretum niorense, 21 espèces. En terme de nombre moyen d'espèces par relevé, les groupements les plus diversifiés sont le groupement végétal à Tamarindus indica et Combretum paniculatum avec 9,45 $\pm 1,25$ espèces et le groupement à Terminalia avicennioides et Ximenia americana avec 12,50 $\pm 1,86$ espèces. Les moins diversifiés sont le groupement à Acacia sieberiana avec 2,84 $\pm 0,39$ espèces et le groupement à $E u$ phorbia sudanica et Combretum niorense avec 4,53 $\pm 0,83$ espèces. Pour ce qui est de l'indice de diversité de Shannon (H) calculé, le groupement à Vitex chrysocarpa et Garcinia livingstonei est plus diversifié $(\mathrm{H}=3,9$ bits). Deux autres groupements sont moins diversifiés à savoir, le groupement à Acacia sieberiana $(\mathrm{H}=1,85)$ et le groupement anthropique à Parkia biglobosa et Piliostigma reticulatum $(\mathrm{H}=$ 2,44). L'indice de Piélou (E) montre qu'un nombre restreint d'espèces imprime la physionomie d'ensemble dans 4 groupements végétaux. Ce sont par ordre d'importance de la valeur de l'indice, le groupement à Vitex chrysocarpa et Garcinia livingstonei $(\mathrm{E}=0,74)$, le groupement à Euphorbia sudanica et Combretum niorense $(\mathrm{E}=0,66)$, le groupement à Burkea africana et Detarium microcarpum $(\mathrm{E}=0,6)$ et le groupement à Acacia gourmaensis et Acacia macrostachya $(\mathrm{E}=0,6)$. Les autres groupements présentent une bonne équitabilité dans la répartition des espèces.

En outre, en s'intéressant à la surface terrière $(G)$, les groupements végétaux ayant les fortes valeurs sont le groupement à Vitex chrysocarpa et Garcinia livingstonei des formations galeries avec $\mathrm{G}=21,3 \pm 6,70 \mathrm{~m}^{2} /$ ha et le groupement végétal à Tamarindus indica et Combretum paniculatum des forêts claires avec $\mathrm{G}=22,57 \pm 3,08 \mathrm{~m}^{2} / \mathrm{ha}$. Les plus faibles valeurs de surface terrière sont observées dans le groupement à Acacia sieberiana des savanes arbustives 
Tableau 2: Caractéristiques floristiques et structurales des groupements végétaux ligneux Table 2: $\quad$ Floristic and structural caracteristics of the communities of woody plants

\begin{tabular}{|c|c|c|c|c|c|c|c|c|c|c|}
\hline Groupement végétal ligneux & Es_Cn & Ba_Dm & Ag_Am & Ta_Xa & Pb_Pr & $\mathrm{Ag} \_\mathrm{Ca}$ & Ti_Cp & TM_Gt & As & VC_GI \\
\hline nombre de relevés & 17 & 24 & 28 & 16 & 12 & 37 & 20 & 26 & 25 & 37 \\
\hline Richesse spécifique (S) & 21 & 35 & 43 & 47 & 29 & 39 & 38 & 36 & 13 & 39 \\
\hline Richesse moyenne par relevés (RM) & $4,53 \pm 0,83$ & $7,83 \pm 0,96$ & $8,46 \pm 2,22$ & $12,50 \pm 1,86$ & $7,42 \pm 1,24$ & $7,19 \pm 0,99$ & $9,45 \pm 1,25$ & $6,35 \pm 0,99$ & $2,84 \pm 0,39$ & $8,38 \pm 0,91$ \\
\hline Indice de Shannon (H) & 2,89 & 3,07 & 3,24 & 3,24 & 2,44 & 2,87 & 2,99 & 2,67 & 1,85 & 3,9 \\
\hline Equitabilité de Piélou€ & 0,66 & 0,6 & 0,6 & 0,58 & 0,5 & 0,54 & 0,57 & 0,52 & 0,5 & 0,74 \\
\hline Surface terrière (G) & $1,38 \pm 0,41$ & $7,11 \pm 1,9$ & $2,83 \pm 0,66$ & $8,53 \pm 1,71$ & $10,69 \pm 3,97$ & $7,11 \pm 1,24$ & $22,57 \pm 3,08$ & $9,12 \pm 1,41$ & $3,03 \pm 1,36$ & $21,3 \pm 6,70$ \\
\hline Densité (D: individus/hectare) & $73,33 \pm 23$ & $250 \pm 50,31$ & $265,56 \pm 50,96$ & $338,89 \pm 50,42$ & $152,22 \pm 42,08$ & $466,67 \pm 102,66$ & $556,67 \pm 131,88$ & $247,78 \pm 69,39$ & $92,22 \pm 25,97$ & $512 \pm 82,46$ \\
\hline Fréquence et recouvrement (\%) & FR-RM (\%) & FR-RM (\%) & FR-RM (\%) & FR-RM (\%) & FR-RM (\%) & FR-RM (\%) & FR-RM (\%) & FR-RM (\%) & FR-RM (\%) & FR-RM (\%) \\
\hline \multicolumn{11}{|l|}{ Espèces } \\
\hline Combretum niorense Aubrév. ex Keay & ||| 3,97 & 00 & 00 & 00 & 00 & 00 & 00 & 00 & 00 & 00 \\
\hline Euphorbia sudanica A. Chev. & $V 5,29$ & | 1,40 & 00 & 00 & 00 & 00 & 00 & 00 & 00 & 00 \\
\hline Ficus abutilifolia (Miq.) Miq. & || 0,56 & 00 & 00 & 00 & 00 & 00 & 00 & 00 & 00 & 00 \\
\hline Ficus ingens (Miq.) Miq. & | 0,03 & 00 & 00 & 00 & 00 & 00 & 00 & 00 & 00 & 00 \\
\hline Gardenia sokotensis Hutch. & | 0,03 & 00 & 00 & 00 & 00 & 00 & 00 & 00 & 00 & 00 \\
\hline Grewia flavescens Juss. & | 0,03 & 00 & 00 & 00 & 00 & 00 & 00 & 00 & 00 & 00 \\
\hline Burkea africana Hook. F. & | 0,03 & V 19,8 & 1 0,36 & | 0,03 & 00 & 00 & 00 & 00 & 00 & 00 \\
\hline Detarium microcarpum G. \& Perr. & 00 & V 10,9 & 1 0,05 & 1 0,03 & 00 & 00 & 00 & 00 & 00 & 00 \\
\hline Annona senegalensis Pers. & 10,03 & IV 5,17 & $\| 0,21$ & $\| 0,78$ & 10,04 & 00 & 00 & 10,02 & 00 & 00 \\
\hline Xeroderris stuhlmannii (Taub.) M.\& E. P. Sousa & 00 & | 0,13 & 00 & 00 & 00 & 00 & 00 & 00 & 00 & 00 \\
\hline Acacia macrostachya Reich. ex Benth. & 00 & 10,06 & ||l 1,04 & 00 & 00 & | 0,01 & 00 & 00 & 00 & 00 \\
\hline Terminalia mollis Laws. & 00 & 00 & 10,02 & 00 & 00 & 00 & 00 & 00 & 00 & 00 \\
\hline Terminalia avicennioides G. \& Perr. & 00 & $\| 0,71$ & 10,02 & V 33,6 & 10,29 & 00 & 00 & 10,02 & 00 & 1 0,08 \\
\hline Strychnos spinosa Lam. & 00 & || 0,21 & | 0,02 & IV 0,66 & 00 & 00 & 00 & 00 & 00 & 00 \\
\hline Ximenia americana L. & 00 & 1 0,04 & | 0,02 & IV 3,03 & $\| 0,13$ & 00 & 00 & | 0,06 & 00 & 00 \\
\hline Maytenus senegalensis (Lam.) Exell. & 00 & 10,02 & | 0,14 & 1110,59 & $\| 0,13$ & 00 & 00 & | 0,06 & 00 & 00 \\
\hline Gardenia erubescens Stapf et Hutch. & 00 & 00 & 1 0,36 & ||| 1,75 & 10,04 & 00 & 00 & 00 & 00 & 00 \\
\hline Gardenia aqualla Stapf \& Hutch. & 00 & 00 & 00 & III 0,38 & 00 & 00 & 00 & | 0,02 & 00 & 00 \\
\hline Dombeya quinqueseta (Planch.) Keay & 00 & | 0,02 & | 0,14 & $\| 2,78$ & 00 & 00 & 00 & 00 & 00 & 00 \\
\hline Lonchocarpus laxiflorus G. et Perr. & 00 & | 0,13 & | 0,05 & \| 0,47 & 00 & | 0,08 & 00 & 00 & 00 & 00 \\
\hline Grewia mollis Juss & 00 & 00 & 1 0,02 & $\| 0,13$ & | 0,04 & 00 & 00 & 00 & 00 & 00 \\
\hline Ziziphus abyssinica Hochst. & 00 & 00 & | 0,05 & \| 0,78 & | 0,04 & | 0,08 & 00 & 00 & 00 & 00 \\
\hline Acacia dudgeoni Craib.ex Holl. & 00 & 00 & 00 & $\| 0,13$ & 00 & 00 & 00 & 00 & 00 & 00 \\
\hline Vitex simplicifolia Oliv. & 00 & 00 & 00 & || 0,31 & | 0,04 & 00 & 00 & 00 & 00 & 00 \\
\hline Hymenocardia acida Tul. & 00 & 00 & 00 & | 0,03 & 00 & 00 & 00 & 00 & 00 & 00 \\
\hline Securidaca longepedunculata Fres. & 00 & 00 & 00 & | 0,41 & 00 & 00 & 00 & 00 & 00 & 00 \\
\hline Parkia biglobosa (Jacq.) Benth. & | 0,18 & 00 & 00 & 00 & V 16,5 & 00 & 00 & 00 & 00 & 00 \\
\hline Prosopis africana (G. et Perr.) Taub. & 00 & 00 & 10,02 & 1 0,06 & IV 1,33 & | 0,01 & 00 & | 0,02 & 00 & | 0,08 \\
\hline Piliostigma reticulatum (Dc.) Hoschst & 00 & 00 & 00 & 00 & III 0,50 & 00 & 00 & | 0,02 & 00 & 00 \\
\hline Combretum sericeum G. Don & 00 & 00 & 00 & 00 & | 0,04 & 00 & 00 & 00 & 00 & 00 \\
\hline Vitellaria paradoxa Gaertn. & 00 & || 1,85 & | 0,05 & V 4,19 & V 15,7 & 00 & 00 & 00 & 00 & 00 \\
\hline Bridelia ferruginea Benth. & 00 & 00 & | 0,02 & | 0,03 & 00 & 00 & 00 & 00 & 00 & 00 \\
\hline Parinari curatellifolia Planch. ex Benth. & 00 & 00 & 00 & 10,03 & 10,25 & 00 & 00 & 00 & 00 & 00 \\
\hline Combretum molle R. Br.ex G. Don & 00 & $\| 0,44$ & $\| 0,27$ & 10,22 & 00 & | 0,05 & 00 & | 0,02 & 00 & 00 \\
\hline Acacia hockii De Wild. & 00 & 10,98 & $\| 0,25$ & | 0,19 & 00 & 00 & 00 & 00 & 00 & 00 \\
\hline Sterculia setigera Del. & 00 & | 0,08 & 10,02 & | 0,03 & 00 & 00 & 00 & 00 & 00 & 00 \\
\hline Pteleopsis suberosa Engl. Et Diels. & 00 & $\| 0,10$ & | 0,04 & \| 0,44 & 00 & 00 & 00 & 1 0,02 & 00 & 00 \\
\hline Combretum micranthum G. Don & | 1,21 & 10,25 & 00 & 00 & 00 & 00 & 00 & 00 & 00 & 00 \\
\hline Terminalia glaucescens Planch. & 00 & | 0,13 & 00 & 00 & 00 & 00 & 00 & 00 & 0,12 & 00 \\
\hline Guiera senegalensis J. F. Gmel. & | 0,06 & 00 & 00 & 00 & | 0,08 & 00 & 00 & 00 & 00 & 00 \\
\hline Terminalia laxiflora Engl. & $\| 0,56$ & $\| 1,65$ & $\| 0,55$ & $\| 11,0$ & $\| 1,17$ & 00 & 00 & 00 & 00 & 00 \\
\hline Combretum glutinosum Perr. ex DC. & ||| 1,35 & IV 6,73 & V 10,5 & ||1 1,75 & V 5,08 & 10,09 & 00 & 10,19 & 00 & 00 \\
\hline Stereospermum kunthianum Cham. & | 0,03 & | 0,48 & 10,02 & $\| 0,28$ & 10,08 & | 0,03 & 10,03 & 00 & 00 & 00 \\
\hline Grewia lasiodiscus K. Schum. & 00 & | 0,04 & | 0,07 & 10,09 & 10,08 & 10,20 & $\| 0,13$ & 10,02 & 00 & 00 \\
\hline Combretum collinum Fresen. & 00 & 10,42 & $\| 0,27$ & ||| 1,13 & ||| 4,75 & || 0,45 & 10,08 & $\| 1,58$ & 00 & | 0,01 \\
\hline Piliostigma thonningii (Schum.) Milne-Redhead & 00 & 10,02 & $\| 0,25$ & ||| 2,88 & 10,25 & 10,05 & 10,03 & IV 4,29 & | 0,12 & | 0,01 \\
\hline Grewia bicolor Juss. & 00 & 10,08 & 1 0,04 & \| 0,13 & 10,04 & | 0,15 & $\| 0,13$ & 00 & 00 & 00 \\
\hline Acacia gourmaensis A. Chev. & 00 & | 0,19 & IV 3,55 & 10,09 & 10,04 & $\| 1,49$ & 10,08 & 1 0,40 & 00 & 00 \\
\hline Lannea acida A. Rich. & ||| 0,53 & $\| 0,88$ & IV 2,50 & 10,03 & 10,04 & 10,09 & 10,05 & 10,52 & 00 & 00 \\
\hline Lannea microcarpa Engl. Et K. Krause & | 0,38 & 10,04 & $\| 1,09$ & | 0,19 & 10,04 & 10,09 & 00 & 10,02 & | 0,92 & 00 \\
\hline Pterocarpus erinaceus Poir. & | 0,79 & $\| 0,44$ & 10,09 & | 1,47 & | 0,04 & | 0,07 & 10,65 & 00 & 10,02 & 00 \\
\hline Sclerocarya birrea (A. Rich.) Hochst. & $\| 0,15$ & 00 & | 0,13 & | 0,19 & 00 & | 0,01 & 00 & 00 & 00 & 00 \\
\hline Dichrostachys cinerea L.Wight \& Arn. & 00 & 10,02 & 1 0,02 & 00 & 00 & 10,05 & 10,08 & 00 & 00 & | 0,01 \\
\hline Crossopteryx febrifuga (Afz. ex G. Don.) Benth. & 00 & 10,08 & ||| 1,13 & IV 7,44 & 00 & | 0,12 & 00 & III 3,25 & 00 & 00 \\
\hline Combretum nigricans Lepr. ex Guill \& Perr & || 0,26 & ||1 0,96 & IV 6,84 & 10,22 & 00 & | 0,88 & V 14,5 & 10,02 & 00 & 00 \\
\hline Bombax costatum Pell. \& Vuill. & | 0,03 & | 0,15 & | 0,02 & | 0,25 & 00 & 00 & 10,15 & | 0,38 & 00 & 00 \\
\hline Mitragyna inermis (Wild.) Kuntze. & 00 & 00 & 00 & 00 & 00 & IV 10,2 & V 17,9 & $\| 0,92$ & V 8,72 & IV 17,53 \\
\hline Flueggea virosa (Roxl. Ex Willd.) Voigt & 00 & 00 & | 0,05 & | 0,03 & | 0,04 & IV 1,32 & 1110,38 & | 0,06 & 00 & 10,27 \\
\hline Daniellia oliveri (R.) Hutch. \& Dalz. & 00 & 00 & 00 & | 0,19 & | 0,04 & | 1,01 & 00 & | 0,90 & 00 & | 0,08 \\
\hline Vitex doniana Sweet. & 00 & 00 & 00 & | 0,03 & | 0,04 & 00 & 00 & | 0,02 & 00 & 00 \\
\hline Combretum fragrans F. Hoffm. & 00 & 00 & 00 & | 0,66 & 00 & ||| 2,93 & 10,68 & IV 5,29 & | 0,54 & | 0,03 \\
\hline Acacia sieberiana DC. & 00 & 00 & 00 & | 0,03 & 00 & 10,16 & 10,18 & ||1 0,31 & V 11 & 10,30 \\
\hline Cadaba farinosa Forsk. & 00 & 00 & 00 & 00 & 00 & 10,09 & 10,03 & 00 & 00 & 00 \\
\hline
\end{tabular}


Continuation du Tableau 2 | Continuation of Table 2

\begin{tabular}{|c|c|c|c|c|c|c|c|c|c|c|}
\hline Groupement végétal ligneux & Es_Cn & $\mathrm{Ba} \_\mathrm{Dm}$ & Ag_Am & Ta_Xa & Pb_Pr & Ag_Ca & Ti_Cp & TM_Gt & As & VC_GI \\
\hline Khaya senegalensis (Desr.) A. Juss. & 00 & 00 & 00 & 00 & 00 & | 0,01 & 11,88 & 00 & 00 & 00 \\
\hline Anogeissus leiocarpus (DC.) Guill. et Perr. & 00 & 10,27 & 10,52 & 00 & 00 & IV 5,59 & $\vee 41,7$ & | 0,06 & 00 & 10,14 \\
\hline Combretum aculeatum Vent & 00 & 00 & | 0,13 & 00 & 00 & IV 3,24 & $\| 0,30$ & 00 & 00 & 00 \\
\hline Acacia gerrardii Benth. & 00 & 00 & 00 & 00 & 00 & IV 23,2 & 10,03 & | 0,13 & | 1,16 & 00 \\
\hline Balanites aegyptiaca (L.) Del. & 00 & 00 & || 0,54 & 10,03 & 00 & ||| 2,43 & $\| 1,00$ & | 0,10 & 00 & I 0,04 \\
\hline Tamarindus indica L. & 00 & 00 & | 0,02 & 00 & 00 & | 0,04 & IV 7,80 & 00 & 00 & 00 \\
\hline Ziziphus mucronata Willd & 00 & 00 & 00 & 00 & 00 & | 0,01 & 1110,38 & 00 & 00 & 00 \\
\hline Combretum paniculatum Vent & 00 & 00 & 00 & 00 & 00 & 00 & 1113,88 & 00 & 00 & | 0,28 \\
\hline Capparis sepiaria L. & 00 & 00 & 00 & 00 & 00 & | 0,11 & $\| 0,38$ & 00 & 00 & 00 \\
\hline Feretia apodanthera Del. & 00 & 00 & 00 & 00 & 00 & | 0,03 & $\| 3,93$ & 00 & 00 & 00 \\
\hline Acacia erythrocalyx brenan & 00 & 00 & 00 & 00 & 00 & 00 & $\| 5,20$ & 00 & 00 & 00 \\
\hline Cassia sieberiana DC. & 00 & 00 & 00 & 00 & 00 & 00 & $\| 1,03$ & 00 & 00 & | 0,61 \\
\hline Diospyros mespiliformis & 00 & 00 & 00 & 00 & 00 & 00 & $\| 1,73$ & 00 & 00 & | 1,19 \\
\hline Asparagus africanus Lam. & 00 & 00 & 00 & 00 & 00 & 00 & 10,03 & 00 & 00 & 00 \\
\hline Keetia cornelia Cham. et Schlecht. & 00 & 00 & 00 & 00 & 00 & 00 & $\| 0,28$ & 00 & 00 & || 2,58 \\
\hline Pseudocedrala kotschyi (Schw.) Harms & 00 & 00 & 10,02 & 00 & 00 & | 0,01 & 10,03 & | 2,44 & 10,02 & 00 \\
\hline Terminalia macroptera G. \& Perr. & 00 & 00 & 00 & 00 & 00 & 1 0,03 & 00 & V 21,6 & | 0,12 & | 0,08 \\
\hline Gardenia ternifolia Schum. Et Thonn. & 00 & 00 & 00 & | 0,19 & 00 & 00 & 10,03 & $\| 0,21$ & 00 & 00 \\
\hline Afzelia africana Sm. & 00 & 00 & 00 & 00 & 00 & 00 & 00 & | 0,02 & 00 & 00 \\
\hline Bridelia scleroneura Müll. Arg. & 00 & 00 & 00 & 00 & 00 & 00 & 00 & 10,02 & 00 & 00 \\
\hline Isoberlinia doka Craib. \& Stapf. & 00 & 00 & 00 & 00 & 00 & 00 & 00 & | 0,02 & 00 & 00 \\
\hline Lannea barteri (Oliv.) Engl. & 00 & 00 & 00 & 00 & 00 & 00 & 00 & | 0,23 & 00 & 00 \\
\hline Crataeva adansonii DC. & 00 & 00 & 00 & 00 & 00 & 00 & 10,08 & 00 & 10,1 & | 0,34 \\
\hline Acacia seyal Del. & 00 & 00 & 00 & 00 & 00 & | 0,03 & 00 & 00 & 10,26 & 00 \\
\hline Borassus aethiopum Mart. & 00 & 00 & 00 & 00 & 00 & 00 & 00 & 00 & | 0,12 & | 1,42 \\
\hline Vitex chrysocarpa Planch. Ex Benth. & 00 & 00 & 00 & 00 & 00 & 00 & 11,90 & 00 & 00 & IV 15,41 \\
\hline Taccazea apiculata Oliv. & 00 & 00 & 00 & 00 & 00 & 00 & 00 & 00 & 00 & IV 0,88 \\
\hline Paulinia pinnata L. & 00 & 00 & 00 & 00 & 00 & 00 & 10,03 & 00 & 00 & ||| 2,09 \\
\hline Cola laurifolia Mast. & 00 & 00 & 00 & 00 & 00 & 00 & 00 & 00 & 00 & ||| 8,14 \\
\hline Garcinia livingstonei T. Anders. & 00 & 00 & 00 & 00 & 00 & 00 & 00 & 00 & 00 & ||| 12,00 \\
\hline Mimosa pigra L. & 00 & 00 & 00 & 00 & 00 & 00 & 00 & 00 & 00 & ||| 6,88 \\
\hline Morelia senegalensis A. Rich. & 00 & 00 & 00 & 00 & 00 & 00 & 00 & 00 & 00 & ||| 9,97 \\
\hline Phyllanthus reticulatus Poir. & 00 & 00 & 00 & 00 & 00 & 10,30 & 00 & 00 & 00 & || 0,68 \\
\hline Combretum acutum Laws. & 00 & 00 & 00 & 00 & 00 & 00 & 00 & 00 & 00 & $\|$ 9,27 \\
\hline Parinari congensis F. Didr. & 00 & 00 & 00 & 00 & 00 & 00 & 00 & 00 & 00 & || 9,39 \\
\hline Syzygium guineense (Willd.) DC. & 00 & 00 & 00 & 00 & 00 & 00 & 00 & 00 & 00 & $\| 11,51$ \\
\hline Ziziphus spina-christi (L.) Desf. & 00 & 00 & 00 & 00 & 00 & 00 & 00 & 00 & 00 & || 5,38 \\
\hline Sarcocephalus latifolius (Smith) Bruce & 00 & 00 & 00 & 00 & 00 & 00 & 10,03 & 10,12 & 00 & 10,54 \\
\hline Antidesma venosum Tul. & 00 & 00 & 00 & 00 & 00 & 00 & 00 & 00 & 00 & | 0,32 \\
\hline Loeseneriella africana (Willd.) Wilczek & 00 & 00 & 00 & 00 & 00 & 00 & 00 & 00 & 00 & 10,26 \\
\hline Pterocarpus santalinoides L'Hér. ex DC. & 00 & 00 & 00 & 00 & 00 & 00 & 00 & 00 & 00 & | 1,19 \\
\hline Rytigynia senegalensis Blume & 00 & 00 & 00 & 00 & 00 & 00 & 00 & 00 & 00 & | 0,04 \\
\hline Triclisia patens oliv. & 00 & 00 & 00 & 00 & 00 & 00 & 00 & 00 & 00 & | 0,35 \\
\hline Uvaria chamae P. Beauv. & 00 & 00 & 00 & 00 & 00 & 00 & 00 & 00 & 00 & | 0,01 \\
\hline Xylopia parviflora (A. Rich.) Hoschst & 00 & 00 & 00 & 00 & 00 & 00 & 00 & 00 & 00 & | 2,70 \\
\hline Adansonia digitata L. & | 0,03 & 00 & 00 & 00 & 00 & | 0,27 & 10,53 & 00 & 00 & 00 \\
\hline
\end{tabular}

Vc_GI: forêts galeries à Vitex chrysocarpa et Garcinia livingstonei / riparian forests of Vitex chrysocarpa and Garcinia livingstonei

As: savanes arbustives marécageuses à Acacia sieberiana / humids wooded grasslands of Acacia sieberiana

Tm_Gt: savanes arborées à Terminalia macroptera et Gardenia ternifolia / open woodlands of Terminalia macroptera and Gardenia ternifolia

Ag_Ca: savanes boisées à Acacia gerrardii et Combretum acuelatum / woodlands of Acacia gerrardii and Combretum acuelatum

Ti_Cp: forêts claires à Tamarindus indica et Combretum paniculatum / woodlands of Tamarindus indica and Combretum paniculatum

Ta_Xa: Savanes arborées à Terminalia avicennioides et Ximenia americana / open woodlands of Terminalia avicennioides and Ximenia americana

Pb_Pr: savanes anthropiques à Parkia biglobosa et Piliostigma reticulatum / anthropogenic savannas of Parkia biglobosa and Piliostigma reticulatum

Ag_Am: savanes arbustives à Acacia gourmaensis et Acacia macrostachya / shrublands of Acacia gourmaensis and Acacia macrostachya

Ba_Dm: savanes arborées à Burkea africana et Detarium microcarpum / open woodlands of Burkea africana and Detarium microcarpum

Es_Cn: savanes arbustives clairsemées à Euphorbia sudanica et Combretum niorense / wooded grasslands of Euphorbia sudanica and Combretum niorense

$\begin{array}{ll}\text { Es_Cn: } & \text { savanes arbustives clairsemées à E } \\ \text { RM: } & \text { récouvrement moyen / mean cover }\end{array}$

$\begin{array}{ll}\text { RM: } & \text { récouvrement moyen / mean cover } \\ \text { FR: } & \text { fréquence relative: / relative fequency: }\end{array}$

$1=$ espèce présente dans moins de 1 à $20 \%$ de relevés / species present in less than 1 to $20 \%$ of the relevés

II = espèce présente dans 21 à $40 \%$ de relevés / species present in 21 to $40 \%$ of the relevés

III = espèce présente dans 41 à $60 \%$ de relevés / species present in 41 to $60 \%$ of the relevés

IV = espèce présente dans 61 à $80 \%$ de relevés / species present in 61 to $80 \%$ of the relevés

Moy. \pm I.C.

Richesse spécifique (S) / species richness

Richesse moyenne par relevés (RM) / mean species richness per relevé $\quad 34 \pm 6,39$

Indice de Shannon $(\mathrm{H})$ / shannon index

Equitabilité de Piélou $€$ / equitability of Piélou

Surface terrière (G) / stand basal area

$7,55 \pm 1,64$
$2,92 \pm 0,33$

$2,92 \pm 0,33$

$0,55 \pm 0,05$

Densité (D: individus/hectare) / Density (D: individuals / hectar)

$9,37 \pm 4,51$
$295,53 \pm 105,89$ 


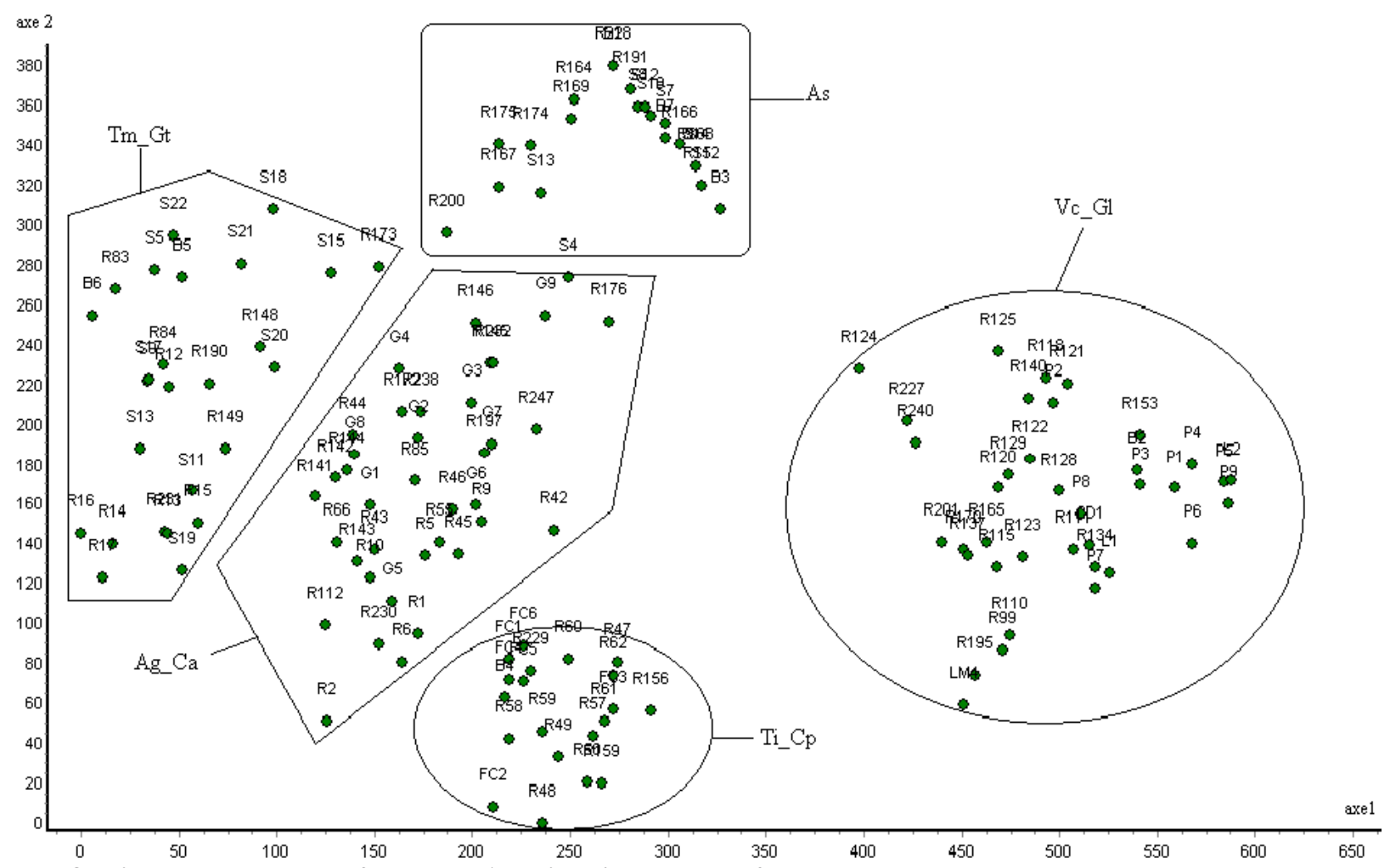

Vc_GI: forêts galeries à Vitex chrysocarpa et Garcinia livingstonei / riparian forests of Vitex chrysocarpa and Garcinia livingstonei

As: $\quad$ savanes arbustives marécageuses à Acacia sieberiana / humids wooded grasslands of Acacia sieberiana

Tm_Gt: savanes arborées à Terminalia macroptera et Gardenia ternifolia / open woodlands of Terminalia macroptera and Gardenia ternifolia

Ag_Ca: savanes boisées à Acacia gerrardii et Combretum acuelatum / woodlands of Acacia gerrardii and Combretum acuelatum

Ti_Cp: forêts claires à Tamarindus indica et Combretum paniculatum / woodlands of Tamarindus indica and Combretum paniculatum

Fig. 3: Discrimination des groupements ligneux des milieux inondés Fig. 3: Discrimination of the woody plant communities of inundated land

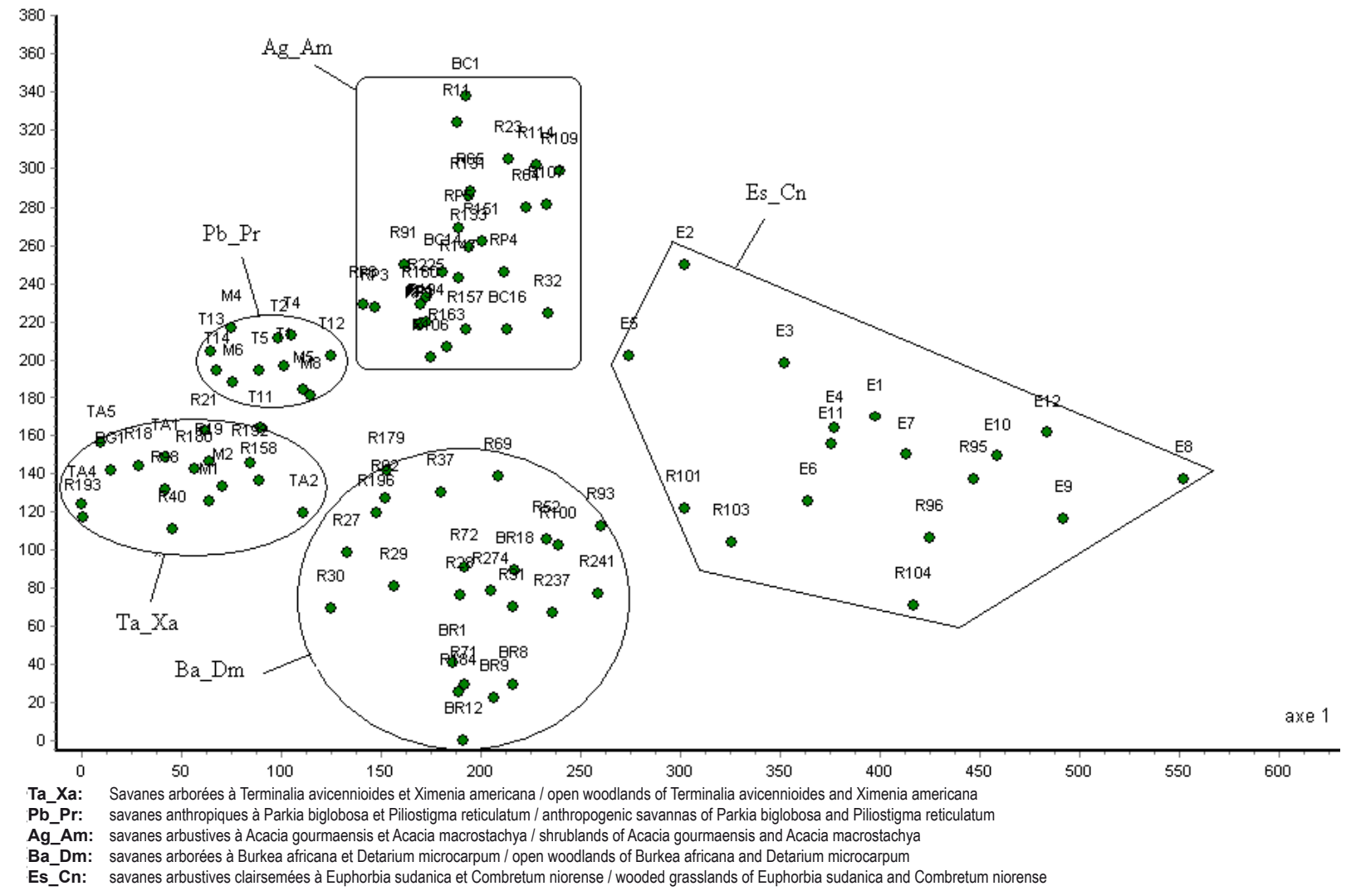

Fig. 4: Discrimination des groupements des milieux exondés

Fig. 4: Discrimination of the woody plant communities of non-inundated land 
marécageuses $\left(\mathrm{G}=3,03 \pm 1,36 \mathrm{~m}^{2} / \mathrm{ha}\right)$, le groupement à Acacia gourmaensis et Acacia macrostachya des formations arbustives clairsemées $\left(\mathrm{G}=2,83 \pm 0,66 \mathrm{~m}^{2} / \mathrm{ha}\right)$ et le groupement à Euphorbia sudanica et Combretum niorense des savanes herbeuses $\left(\mathrm{G}=1,38 \pm 0,41 \mathrm{~m}^{2} / \mathrm{ha}\right)$. Parallèlement à la surface terrière ces trois derniers groupements sont celles qui sont les moins denses. Ceux qui sont les plus denses sont le groupement à Vitex chrysocarpa et Garcinia livingstonei $(\mathrm{D}=512 \pm 82,46$ individus $/ \mathrm{ha})$, le groupement végétal à Tamarindus indica et Combretum paniculatum (D = 556,67 $\pm 131,88$ individus /ha) et le groupement à Acacia gerrardii et Combretum acuelatum des savanes boisées $(\mathrm{D}=466,67 \pm$ 102,66 individus /ha). Les autres groupements décrivent des savanes arborées.

\subsection{Structure en classe de diamètre des groupements végétaux}

En considérant la répartition en classe de diamètre des peuplements de chaque groupement, on note une variabilité de structure (fig. 5). La densité des individus est faible pour les savanes arbustives marécageuses à Acacia sieberiana et les savanes arbustives clairsemées à Euphorbia sudanica et Combretum niorense quelque soit la classe de diamètre considérée. Dans les autres groupements, la densité des individus est élevée surtout pour les deux premières classes de diamètre $(5-10 \mathrm{~cm}, 10-15 \mathrm{~cm})$. C'est dans les forêts galeries à Vitex chrysocarpa et Garcinia livingstonei, les forêts claires à Tamarindus indica et Combretum acuelatum et les savanes anthropiques à Parkia biglobosa et Piliostigma reticulatum qu'on rencontre un nombre important des individus de gros diamètres (diamètre $\geq 50 \mathrm{~cm}$ ). L'analyse de la structure démographique des différents groupements végétaux montre une tendance globale où le nombre d'individus est plus éle- vé dans les petites classes de diamètres et diminue dans les classes de diamètres supérieurs. Cette tendance se traduit par une forme de la structure démographique en L. Néanmoins, le groupement à Parkia biglobosa et Piliostigma reticulatum décrit une structure assez particulière marquée par un faible nombre des individus de classe de diamètre intermédiaire et un nombre proportionnellement élevé des classes des individus de gros diamètre $(45-50 \mathrm{~cm}, \geq 50 \mathrm{~cm})$.

\subsection{Relation diversité - structure et détermination des groupes homogènes}

Le test de corrélation de Pearson calculé à $\mathrm{p}<0,5 \%$ montre qu'il y a une liaison entre les différents paramètres de la variable diversité et de la variable structure. Les paramètres les plus corrélés aux autres sont la richesse spécifique totale et l'indice de diversité de Shannon (tableau 3) d'une part et d'autres part la densité et la surface terrière. Toutefois les valeurs de corrélation les plus élevées s'observent au sein des paramètres d'une même variable. Les résultats du test LSD à $\mathrm{p}<5 \%$ ont permis de rassembler les groupements végétaux en groupes homogènes (différence non significative) pour chaque paramètre du tableau 4 . On distingue trois groupes pour la richesse moyenne par relevé (RM1, RM2, RM3) et trois groupes pour la densité (D1, D2, D3) et pour la surface terrière (G1, G2, G3). Parmi les groupements végétaux distingués, quatre illustrent nettement la relation de corrélation entre la diversité et la structure. Ainsi, les groupements végétaux à Tamarindus indica et Combretum paniculatum et le groupement à Vitex chrysocarpa et Garcinia livingstonei dans une moindre mésure décrivent les formations les plus denses (D3) et ayant les surfaces terrières les plus grandes (G3) font partir également des formations plus diversifiées (RM3, RM2). Aussi le groupement à Euphorbia sudanica et

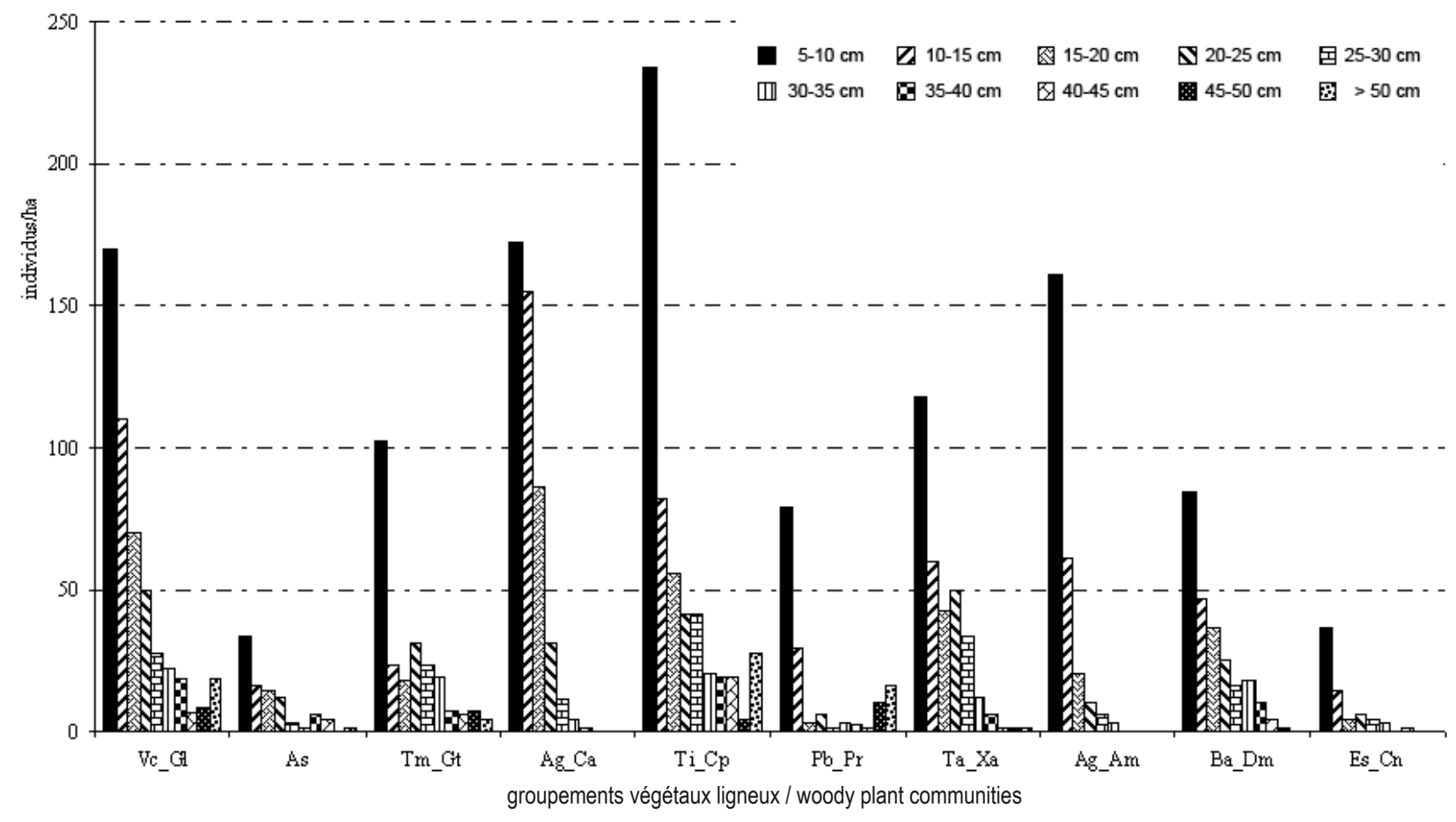

Fig. 5: Structure demographique en classes de diamètre des groupe- $\quad$ Fig. 5: Pattern of size class distribution of the communities of woody ments ligneux plants 
Combretum niorense et celui à Acacia sieberiana sont les formations clairsemées (D1) à surfaces terrières très faibles (G1) et sont moins diversifiés en espèces (RM1).

\section{Discussion}

\subsection{Les groupements végétaux}

Floristiquement, la plupart des groupements végétaux ligneux que nous avons déterminés ont été déjà décrits par certains auteurs. Le groupement à Vitex chrysocarpa et Garcinia livingstonei des lits mineurs des cours d'eau correspond au groupement à Combretum acutum et Garcinia livingstonei décrit par NATTA (2003) sur la rivière Pendjari.

Delvingt et al. (1989) ont fait une description de la flore du parc national de la Pendjari au Bénin qui est contigu à celui d'Arly. Ils y ont décrits des associations végétales qui peuvent être assimilées à certains de nos groupements identifiés en tenant compte des affinités écologiques et floristiques. Ainsi l'association à Acacia sieberiana et Mitragyna inermis des sols engorgés décrits dans la Pendjari correspondrait au groupement à Acacia sieberiana des plaines d'inondation décrits dans cette étude. L'association à Terminalia macroptera et Acacia sieberiana sur les plaines alluviales plus ou moins drainées constitue également le groupement à Terminalia macroptera et Gardenia ternifolia distingués dans notre cas. Par ailleurs, OuÉDRAOGO (2004) et OUOBA (2006)

Tableau 3: Valeurs de corrélation de Pearson $(p<0,5 \%)$ entre paramètres de diversité et de structure

Table 3: Values of Pearson correlation $(p<0,5 \%)$ between diversity and structure parameters

\begin{tabular}{l|cccccc}
\hline Variables & S & RM & H & E & G & D \\
\hline S & 1 & & & & & \\
RM & $0,90^{*}$ & 1 & & & & \\
H & $0,73^{*}$ & $0,65^{*}$ & 1 & & & \\
E & 0,24 & 0,21 & $0,83^{*}$ & 1 & & \\
G & 0,38 & 0,46 & 0,47 & 0,30 & 1 & \\
D & $0,69^{*}$ & 0,61 & 0,63 & 0,32 & $0,79^{*}$ & 1 \\
\hline
\end{tabular}

S: richesse totale / total richness, RM: richesse moyenne par relevé / richness per relevé, H: indice de Shannon / Shannon index, E: Indice de Piélou / Piélou Evenness,

G: surface terrière / basal area, D: densité / density.

*Valeurs significatives / significant correlation

Tableau 4: Classification des groupements en groupes homogènes en fonction de 3 paramètres

Table 4: Classification of the plant communities in homogenous groups according to 3 parameters

\begin{tabular}{lccc}
\hline Groupements végétaux ligneux & $\begin{array}{c}\text { Richesse } \\
\text { moyenne } \\
\text { par relevé }\end{array}$ & Densité & $\begin{array}{c}\text { Surface } \\
\text { terrière }\end{array}$ \\
\hline Vitex chrysocarpa et Garcinia livingstonei & $\mathrm{RM} 2$ & $\mathrm{D} 3$ & $\mathrm{G} 3$ \\
Tamarindus indica et Combretum paniculatum & $\mathrm{RM} 3$ & $\mathrm{D} 3$ & $\mathrm{G} 3$ \\
Acacia gerrardii et Combretum acuelatum & $\mathrm{RM} 2$ & $\mathrm{D} 3$ & $\mathrm{G} 2$ \\
Terminalia macroptera et Gardenia ternifolia & $\mathrm{RM} 2$ & $\mathrm{D} 2$ & $\mathrm{G} 2$ \\
Acacia sieberiana & $\mathrm{RM} 1$ & $\mathrm{D} 1$ & $\mathrm{G} 1$ \\
Terminalia avicennioides et Ximenia americana & $\mathrm{RM} 3$ & $\mathrm{D} 2$ & $\mathrm{G} 2$ \\
Parkia biglobosa et Piliostigma reticulatum & $\mathrm{RM} 2$ & $\mathrm{D} 1$ & $\mathrm{G} 2$ \\
Acacia gourmaensis et Acacia macrostachya & $\mathrm{RM} 2$ & $\mathrm{D} 2$ & $\mathrm{G} 1$ \\
Burkea africana et Detarium microcarpum & $\mathrm{RM} 2$ & $\mathrm{D} 2$ & $\mathrm{G} 2$ \\
Euphorbia sudanica et Combretum niorense & $\mathrm{RM} 1$ & $\mathrm{D} 1$ & $\mathrm{G} 1$ \\
\hline
\end{tabular}

Les mêmes sigles désignent l'absence de significativité entre les groupements.

Les chiffres 1, 2, 3 signifient respectivement valeurs faibles aux plus élevées pour chaque paramètre.

The same acronyms show that there is no significant difference between woody plant communities

The numbers 1, 2, 3 stand for respectively low to high values for each parameter ont décrit au Sud-Ouest du Burkina Faso respectivement dans la forêt classée de Bansié et la forêt classée de Niangoloko, des groupements à Terminalia macroptera qui évoluent sur des sols limono-argileux. Cela dénote que c'est un groupement largement répandu dans la région soudanienne du Burkina Faso.

AKoÈGNINOU et al. (2006) ont mentionné toujours dans le Parc national de la Pendjari, la présence des savanes arborées à Acacia gerrardii. Ces unités à Acacia gerrardii correspondent sûrement au groupement à Acacia gerrardii et Combretum acuelatum décrit dans le parc d'Arly. En se référant aux travaux de MONSCH et VAN DER MAESEN (2004) et de Wittig et al. (2004) portant sur la taxonomie et la distribution du genre Acacia au Burkina Faso, l'existence d'Acacia gerrardii n'a pas été signalée ce qui fait que le groupement à Acacia gerrardii et Combretum acuelatum reste assez spécifique à cette région Est du pays à cause de l'espèce caractéristique Acacia gerrardii. Toutefois, THIOMBIANO (2005) a décrit un groupement à Combretum acuelatum localisé dans le secteur sud-sahelien sur des sols argileux et gravillonnaires dont plus de la moitié des espèces font partie du cortège floristique du groupement à Acacia gerrardii et Combretum acuelatum.

Le groupement végétal à Tamarindus indica et Combretum paniculatum ressemble d'une part du point de vue écologique et physionomique, au groupement forestier à Anogeissus leiocarpus identifié par OUOBA (2006) où Anogeissus leiocarpus et Feretia apodenthera caractérisent bien ce groupement et y sont dominantes respectivement dans la strate arborée et dans la strate arbustive. Dans le groupement à Tamarindus indica et Combretum paniculatum, on remarque cette même dominance de ces deux espèces (tableau 2). Cependant Anogeissus leiocarpus n'y est pas caractéristique. D'autre part ce groupement à Tamarindus indica et Combretum paniculatum sur le plan floristique serait une variante plus diversifiée du groupement à Combretum paniculatum décrit par THIOMBIANo (2005) dans le secteur phytogéographique sud-sahélien.

Le groupement à Terminalia avicennioides et Ximenia americana ressemble fortement au groupement à Terminalia avicennioides du supra-groupement à Vitellaria paradoxa que Thiombiano (2005) a définit dans le même secteur phytogéographique (nord soudanien) sur des sols sablo-limoneux et limono-argileux. Les deux groupements sont caractérisés a peu près par les mêmes espèces à savoir Terminalia avicennioides, Maytenus senegalensis, Strychnos spinosa, Vitex simplicifolia.

Le groupement à Parkia biglobosa et Piliostigma reticulatum et le groupement à Terminalia avicennioides et Ximenia americana partagent les mêmes affinités écologiques et ont une grande similarité floristique. Leur différence réside dans le mode d'exploitation du milieu. Le groupement à Parkia biglobosa et Piliostigma reticulatum occupe un espace précis du parc qui s'étend en une bande continue d'environ $500 \mathrm{~m}$ de largeur en moyenne sur une distance de $20 \mathrm{~km}$ de long dans la partie périphérique sud-ouest du parc. Cet espace constitue les anciens champs des populations riveraines. Les zones anthropisées environnantes du parc, ne décrivent dans leur ensemble que des parcs arborées dominées par Parkia biglobosa et Vitellaria paradoxa. Ce sont ces 
deux espèces qui dominent également dans le groupement anthropique à Parkia biglobosa et Piliostigma reticulatum dans le parc (tableau 2). WALA (2004) mentionne la présence de groupement anthropophile à Vitellaria paradoxa et Parkia biglobosa des jachères de longues durée sur la chaîne de l'Atakora au Bénin. En effet, le groupement à Parkia biglobosa et Piliostigma reticulatum constitue également des anciennes jachères dont la flore revèle la marque d'activités anthropiques passées. distingués dans le parc n'est en réalité que. En considérant les affinités floristiques et écologiques, ce groupement ne constitue en réalité dans une série régressive, un stade du groupement à Terminalia avicennioides et Ximenia americana.

Le groupement à Acacia gourmaensis et Acacia macrostachya évoluant sur les sols gravillonnaires des plateaux peut rassembler deux types de végétation ayant des liens phytoécologiques étroits à savoir le type de végétation à Combretum molle et celui à Combretum nigricans que HAHNHADJALI (1998) a déterminé dans la même région. Combretum molle reste caractéristique dans le groupement à Acacia gourmaensis et Acacia macrostachya. En revanche, Combretum nigricans est plutôt fréquente.

Enfin, Les groupements à Burkea africana et Detarium microcarpum et celui à Euphorbia sudanica et Combretum niorense décrit sur la portion de la chaîne de montagne du Gobnangou du parc d'Arly et également sur les autres buttes gréseuses, ont été déjà décrits par d'autres auteurs antérieurement (WiTTIG et al. 2000, OUÉDRAOGO 2006) dans leurs travaux sur la flore de la chaîne du Gobnangou.

\subsection{Les gradients de variation et la variabilité flori- stique des groupements}

La structure des données floristiques établit dans les plans factoriels (fig. 3 et 4 ) sur la base de la méthode DCA permet de rendre compte du changement de la composition floristique suivant la diversité des habitats dans le parc d'Arly. Le premier axe (axe 1) avec toujours la valeur propre la plus élevée et la longueur de gradient la plus importante (tableau 1) décrit le gradient topographique comme principal facteur environnemental qui contrôle la distribution des groupements ligneux. En général pour les régions tropicales où la variation altitudinale est faible c'est la topographie qui est toujours citée comme variable discriminante à laquelle on corrèle le type de sol (SINSIN 1993) ou l'humidité du sol (WALA 2004). En outre, la longeur des gradients exprime aussi la ß-diversité (WILSON et al. 1984). Dans le cas de notre analyse globale, la longueur du gradient de variation suivant l'axe 1 (fig. 2) est relativement grande $(9,36)$. Cela illustre la grande variabilité floristique et celle des écosystèmes dans le parc national d'Arly. Neanmoins, la variabilité floristique diminue lorsqu'on effectue des analyses partielles. La différence floristique entre les groupements végétaux des milieux d'inondations est légèrement plus importante qu'entre les groupements des milieux exondés en considérant les longueurs de gradient (tableau 1). C'est la spécificité de certains écosystèmes qui expliquent la grande variabilité floristique des communautés végétales. En effet dans les milieux inondés, les galeries forestières constituent un écosystème spécial aussi bien par les conditions écolo- giques avec la présence presque permanente de l'eau, que par la flore (NATTA 2003). Parmi les groupements distingués, celui à Vitex chrysocarpa et Garcinia livingstonei des formations galeries renferment plus d'espèces exclusives au groupement (tableau 2). Ce sont ces espèces exclusives qui contribuent plus à la discrimination des groupements dans les analyses multifactorielles des structures de données floristiques. Il y aussi dans les milieux exondés, des habitats spéciaux tels que les buttes rocheuses et la chaîne de montagne du Gobnangou qui sont colonisées par le groupement à Burkea africana et Detarium microcarpum et le groupement à Euphorbia sudanica et Combretum niorense. Cependant, plusieurs espèces ligneuses des milieux exondés ont une amplitude écologique plus grande, ce qui se traduit par une grande homogénéité floristique des paysages et un nombre restreint d'espèces exclusives aux groupements de ces milieux.

\subsection{Structure}

Les structures en L des peuplements des différents groupements ligneux décrits traduisent des formations stables (OUÉDRAOGO 2006). Cette structure est caractérisée sur la base de la répartition des individus en classe de diamètre, par un grand nombre de petits individus, un petit nombre de grands individus et une réduction régulière du nombre d'individus d'une classe de dimension à la suivante (SAMBOU 2004). Les groupements présentent globalement cette tendance et pour chacun d'entre eux, la struture demographique resulte de la contribution des individus des différentes espèces constitutives du groupement. L'équilibre de ces différentes unités de formations revèlent leur capacité d'adaptation aux pressions du milieu, notamment les feux précoces ainsi que les conditions édaphiques.

Par ailleurs, la structure du groupement anthropique à Parkia biglobosa et Pilostigma reticulatum présente des signes de déséquilibre du fait que les individus de classes de diamètres intermédiaires sont faiblement représentés. Parkia biglobosa et Vitellaria paradoxa constituent l'essentiel de l'effectif des individus de gros diamètres (diamètre $\geq 50$ $\mathrm{cm}$ ) dans le groupement qu'elles décrivent. Dans le parc, l'association de ces deux espèces dans les endroits tardivement classés est témoin des traces anthropiques passées. Ces deux espèces sont souvent entretenues dans les agrosystèmes ou plantées (cas de Parkia biglobosa) comme des espèces utilitaires et sont reconnues comme des espèces fréquentes dans les parcs agroforestiers soudaniens (OUÉDRAOGO 1995, BERnARD et al. 1996, Depommier et al. 1996). Cependant l'évolution de ce groupement vers un stade plus équilibré est soutenue par la recrudescence de jeunes individus (fig. 5). Cette tendance évolutive montre la possibilité de restauration de la biodiversité d'un milieu en absence de pressions anthropiques et également du rôle des réserves dans la conservation durable de la biodiversité.

\subsection{Relation entre diversité, structure et facteurs écolo- giques}

La diversité floristique généralement dépend des facteurs environnementaux tels que les conditions de sol et des facteurs de dynamique comme le feu (BACKEUs et al. 2006). 
L'arbre est un agent biologique qui agit sur les propriétes physico-chimiques du sol à travers son système racinaire par les échanges minéraux, la production de litière par la chute des feuilles ou des parties mortes (SMIT 2004). Dans ce sens, l'influence de la végétation sur la fertilité du sol serait plus marquée dans les types de formations les plus denses et plus couvertes c'est-à-dire dans les forêts claires à Tamarindus indica et Combretum paniculatum et les forêts galeries à Vitex chrysocarpa et Garcinia livingstonei avec respectivement des densités de 556,67 et 512 individus à l'hectare et des taux de recouvrement moyen des arbres de $71,67 \%$ et $65 \%$ (tableau 2). Au niveau des structures démographiques de ces deux groupements ligneux, on peut remarquer une bonne rejuvénisation des peuplements à travers l'importance des individus des classes de diamètres inférieurs (fig. 5). Cela indique que les sols y herbergent une certaine fertilité favorable à cette dynamique et aussi au développement de plusieurs espèces ligneuses. Ces formations forestières sont également peu affectées par les feux précoces d'aménagement effectués périodiquement dans le parc, en raison du faible développement du tapis herbacé (OUOBA 2006). Ainsi, l'impact modéré ou faible du feu sur ces types de formations favorise également leur diversification et se traduit par une progression vers une végétation forestière (Houinato 2001).

Concernant les deux groupements moins diversifiés, la position topographique et la nature du sol ne favorisent pas une colonisation des milieux par plusieurs espèces. Le groupement à Acacia sieberiana est non seulement pauvre en espèces mais sur le plan structure est moins dense et a une surface terrière très faible. Dans ces savanes arbustives marécageuses, l'eau stagne pendant la période favorable à la végétation c'est à dire du début de la saison pluvieuse jusqu'au tiers de la saison sèche et le sol est fortement argileux. Ces conditions sont asphyxiantes et très sélectives pour l'implantation et l'épanouissement de plusieurs espèces. Seules Acacia sieberiana et Mitragyna inermis y sont fréquentes. Les autres espèces du groupement sont très rarement rencontrées (tableau 2). C'est dans ces écosystèmes qu'on enregistre le plus faible nombre d'espèces de la classe de diamètre $5-10 \mathrm{~cm}$ proche de la population juvénile. Egalement à certains endroits sur la chaîne de montagne du Gobnangou et sur les buttes rocheuses occupées par le groupement à Euphorbia sudanica et Combretum niorense où le sol est fortement induré (milieu sélectif), la diversité floristique est basse et la structure de la végétation clairsemée. Les milieux moyennement diversifiés restent ceux qui renferment les conditions topographiques intermédiaires à savoir les plateaux et les systèmes de glacis.

Dans une étude ayant pour objectif de montrer l'influence des nutriments du sol sur la structure et la diversité des groupements végétaux, WIJESINGHE et al. 2005 à travers des expérimentations ont montré que l'apport du NPK suivant plusieurs scénarios affectait plutôt la structure (la productivité) des groupements que leur diversité. L'effet non significatif des traitements sur la diversité spécifique que ces auteurs ont observé, peut s'expliquer par la large plasticité écologique des espèces expérimentales, au nombre limité de paramètre chimiques du sol utilisés ainsi qu'aux conditions physiques invariables du sol.
Les conditions de sol demeurent les principaux facteurs écologiques qui contrôlent à la fois la diversité et la structure des formations ligneuses dans notre site d'étude où les conditions climatiques sont homogènes.

\section{Conclusion}

Les analyses phytosociologique et dendrométrique de la végétation ligneuse du parc national d'Arly ont montré qu'il existe une corrélation entre la diversité, la structure et les conditions écologiques colonisées par les différents groupements végétaux discriminés. La structure de la végétation qui est une résultante des conditions topographiques et édaphiques est également une expression de la diversité d'un milieu. Ainsi, les formations les plus denses et à surface terrière élevée sont plus diversifiées. Par ailleurs, les formations moins denses et à valeurs de surface terrière basses sont les plus pauvres en espèces. Ces informations pourraient être utilisées pour estimer la diversité des écosystèmes de l'ensemble des aires protégées de la région d'étude à partir des méthodes de télédétection se référant essentiellement à la structure physionomique dans l'analyse de la végétation. En outre, il ressort à partir de l'analyse de la structure démographique suivant les classes de diamètre que les peuplements des différents groupements végétaux décrivent une stabilité. Cette tendance étant globale, il sérait important pour une analyse encore plus fine de la végétation, d'étudier la structure en classe de diamètre pour chaque espèce ligneuse en vue de déterminer celles qui ont une dynamique compromise.

\section{REMERCIEMENTS}

Nos remerciements vont à l'endroit du projet BIOTA Frankfurt financer par le BMBF (FKZ: 01 LC 0617D1) pour les soutiens financiers et matériels, également à toute personne ayant contribué à la publication de cet article.

\section{BiBLIOGRAPHIE}

AdOMOU, A.C. (2005): Vegetation patterns and environmental gradients in Benin: implications for biogeography and conservation. PhD thesis, Wageningen University, Netherlands, $133 \mathrm{p}$.

Akoègninou, A., Van der Burg, W.J., VAn der MAesen, L.J.G. (2006): Flore analytique du Bénin. Backhuys Publishers, $1020 \mathrm{p}$.

BACKéus, I., BöRge, P., Lennart, S., Ruffo, C. (2006): Tree community and structural dynamics in miombo (Brachstegia-Julbernardia) woodland, Tanzania. Forest Ecol. Management 230: 171-178.

Bernard C., Ouattara N., Peltier R. (1996): Place du parc à Faidherbia albida dans un terroir soudanien, le cas d'un village sénoufou au nord de la Côte d'Ivoire. In les parcs à Faidherbia: Cahiers scientifiques CIRAD-Forêt: 173-189.

Boulet R., Leprun J.C. (1969): Carte pédologique de reconnaissance de la République de Haute Volta, Feuille Est au 1/500 000 ORSTOM, Dakar.

Condit R., Sukumar R., Hubbell P.S., Foster, R.B. (1998): Predicting Population Trends from Size Distributions: A Direct Test in a Tropical Tree Community. American Naturalist 152: 495-509. 
Couteron P., KoKou K. (1995): Contribution à la connaissance de la végétation du parc nationale de la Comoé. Rapport, Dir.générale des eaux et forêts (Côte d'Ivoire), 34 p.

Delvingt, W., Heymans J., C., Sinsin, B. (1989): Guide du Parc national de la Pendjari. AGRER, 125 p.

Depommier, D., Detienne, P. (1996): Croissance de Faidherbia albida dans les parcs du Burkina Faso. In les parcs à Faidherbia: Cahiers scientifiques CIRAD-Forêt: 23-42.

Feoli, E., Ferro, G., Ganis, P. (2006): Validation of phytosociological classifications based on a fuzzy set approach. Community Ecol. 7: 99-108.

Goudiaby, A., Sambou, B., BÂ, A.T., Mвow, C. (2004): La structure de la forêt galerie de la vallée de la cascade de Dindéféllo, sud-est du Sénégal. Actes de l'atelier de Fada N'Gourma (Burkina Faso): 203-223.

GuinKo, S. (1984): La végétation de la Haute Volta. Thèse de doctorat d'Etat, Université de Bordeaux III, 394 p.

Guinochet, M. (1973): Phytosociologie. Ed. Masson \& Cie, Paris, $227 \mathrm{p}$.

HAHN-Hadjali, K. (1998): Les groupements végétaux des savanes du sud-est du Burkina Faso (Afrique de l'ouest). Etudes flor. Vég. Burkina Faso 3: 3-79.

Houinato, M.R.B. (2001): Phytosociologie, écologie, production et capacité de charge des formations végétales paturées dans la région des monts Kouffé (Bénin). Thèse de doctorat, Université Libre de Bruxelles, Belgique, 255 p.

JON F., JON, C.L. (1997): Biodiversity and environmental stability. Biodiversity and Conservation 6: 315-323.

JÜrgens, N., Strohbach, B. Akhtar-Schuster, M., AuStermüHLe, R., Becker, T., Hachfeld, B., Schmiedel, U., Strohbach, M. (2003): Changes in botanical biodiversity with regard to changes in land use practices and climate: standardised monitoring and transect analysis. In German programm on biodiversity and global change (phase I, 20002004): Status report: 98-99.

KÉvin, M.G., SAM, C., SusAn, S. (2000): Multivariate statistics for wildlife and ecology research. Ed. Springer, 283 p.

Monsch, A.K., Van Der Maesen, L.J.G. (2004): Une étude taxonomique des Acacia (Mimosoideae) du Burkina Faso. Etudes flor. Vég. Burkina Faso 8: 3-18.

NATTA, A.K. (2003): Ecological assessment of riparian forests in Benin: phytodiversity, phytosociology, and spatial distribution of tree species. PhD thesis, Wageningen University, Netherlands, $222 \mathrm{p}$.

OuÉDraogo, A.S. (1995): Parkia biglobosa (Leguminosae) en Afrique de l'Ouest: Biosystématique et Amélioration. Thèse de doctorat, Université de Wageningen, 205 p.

OuÉDraogo, A. (2006): Diversité et dynamique de la végétation ligneuse de la partie orientale du Burkina Faso. Thèse de doctorat, Université de Ouagadougou, 196 p.

OuÉDraogo, O. (2004): Etude de la phytodiversité de la forêt classée de Bansié (Zone sud-soudanienne du Burkina Faso). DEA, Université de Ouagadougou, 76 p.

Oumorou, M. (2003): Etude écologique, floristique, phytogéographique et phytosociologique des inselbegrs du Bénin. Thèse de doctorat, Université Libre de Bruxelles, Belgique, $231 \mathrm{p}$.

Ouoba, P. (2006): Flore et végétation de la forêt classée de Niangoloko, Sud-Ouest du Burkina Faso. Thèse de doctorat, Université de Ouagadougou, 144 p.
SAmbou, B. (2004): Evaluation de l'état, de la dynamique et des tendances évolutives de la flore et de la végétation ligneuses dans les domaines soudanien et sub-guinéen au Sénégal. Thèse de doctorat, UCAD Dakar, 210 p.

Sinsin, B. (1993): Phytosociolgie, écologie, valeur pastorale, production et capacité de charge des pâturages du périmètre Nikki-Kalalé au Nord-Bénin. Thèse de doctorat, Université Libre de Bruxelles, Belgique, $350 \mathrm{p}$.

SMIT, G.N. (2004): An approach to tree thinning to structure southern African savannas for long-term restoration from bush encroachment. J. Environmental Management 71: 179-191.

Thiombiano, A. (2005): Les Combretaceae du Burkina Faso: Taxonomie, écologie, dynamique et régénération des espèces. Thèse de doctorat d'Etat, Université de Ouagadougou, $271 \mathrm{p}$.

UICN (2004): Gestion participative et exploitation durable de la biodiversité dans l'Est du Burkina Faso. Rapport, 21 p.

WALA, K. (2004): La végétation de la chaîne de l'Atakora au Bénin: diversité floristique, phytosociologie et impact humain. Thèse de doctorat, Université de Lomé, 139 p.

WiJesinghe, K.D., John, E.A., Hutchings, M.J. (2005): Does pattern of soil resource heterogeneity determine plant community structure? An experimental investigation Ed. Ecological Society, J. Ecol. 93: 99-112.

Wilson, M.V., SchmidA, A. (1984): Measuring Beta diversity with presence-absence data. J. Ecol. 72: 1055-1064.

Wittig, R., Hahn-Hadjali, K., Thiombiano, A. (2000): Les particularités de la vegetation et de la flore de la chaîne du Gobnangou dans le Sud-Est du Burkina Faso. Etudes flor. Vég. Burkina Faso 5: 49-64.

Wittig, R., Schmidt, M., Thiombiano, A. (2004): Cartes de distribution des espèces du genre Acacia L. au Burkina Faso. Etudes flor. Vég. Burkina Faso 8: 19-26.

\section{Adresses des auteurs:}

Oumarou Ouedraogo Adjima Thiombiano Sita Guinko

Laboratoire de Biologie et Ecologie Végétales Unité de Recherches en Sciences de la Vie et de la Terre (UFR/SVT)

Université de Ouagadougou

03 BP 7021 Ouagadougou 03

Burkina Faso

eMail:

ouedraogooumar@yahoo.fr

\section{Karen Hahn-Hadjali}

Institute for Ecology, Evolution and Diversity

J. W. Goethe-University

Siesmayerstr. 70

60323 Frankfurt/M

Allemagne

\section{eMail:}

hahn-hadjali@bio.uni-frankfurt.de 